\title{
Hamann und Kant.
}

\author{
Von W. Lügert in Halle a. S.
}

Das Verhältnis von Hamann und Kant ist darum so interessant und für beide Männer charakteristisch, weil sie von ihrer innerlichsten geistigen Art bis hinaus in die kleinsten Äusserlichkeiten so grundverschieden sind. Ds ist darum für das Verständnis beider Männer lehrreich, sie im Verkehr mit einander zu beobachten. Die äusseren und inneren Beziehungen zwischen Hamann und Kant hat Dr. Heinrich Weber in gründlicher und erschöpfender Weise durchforscht, und dabei die mannigfachen schiefen Urteile über das Verhältnis der beiden Männer zu einander korrigiert. 1)

Zunächst werden die äusseren Berührungen zwischen beiden dargestellt. Freilich erfährt schon das erste Ergebnis (Seite 17: „das erste Beisammensein, das Hamann der Erwähnung wert hielt, fand vielmehr Anfang Juli 1759 statt $^{\text {() }}$ eine Korrektur durch eine andere wertvolle Arbeit Webers, nämlich durch die Herausgabe bisher ungedruckter Briefe Hamanns aus dem HamannNachlass Friedrich Roths. Dieses höchst verdienstliche und für die Kenntnis Hamanns wichtige Buch kommt indess für einen Aufsatz in dieser Zeitschrift nur insofern in Betracht, als es neues Licht auf den Verkehr zwischen Hamann und Kant wirft.2) Aus den Briefen, von denen Weber erst gehört hatte, als er seine erste Schrift bereits dem.Druck übergeben hatte, geht nun hervor, dass Kant und Hamann schon in Juli 1756 zusammentrafen (Seite 36). Ein weiteres Zusammensein : wird im August gemeldet

1) Vgl. zum folgenden Aufsatz: H. Weber, Hamann und Kant. München 1904. - Derselbe, Neue Hamanniana. München 1905. R. Unger, Hamanns Sprachtheorie im Zusammenhange seines Denkens. München 190う.

2) Neue Notizen über Kant, die besonders auch die Prolegomena betreffen, sind zusammengestellt S. 129-135. 
(Seite 37). Danit ist also die von Weber zurückgewiesene Annahme Gildemeisters, dass schon in den fünfziger Jahren ein Verhältnis zwischen beiden stattgefunden habe, bestätigt.

Die erste nähere Berührung zwischen Kant und Hamann ist gleich für beide Männer charakteristisch. Sie wird vermittelt durch den gemeinsamen Freund Berens. Dem war die Veränderung, die mit Hamann durch seine "Bekehrung: in London vorgegangen war, unheimlich, und Kant sollte ihm helfen, Hamann zur Vernunft zu bringen. Kant besuchte ihn mit Berens und versuchte, ihn „zur Mitarbeit an populärer Aufklärung zu gewinnen“. Die Folge ist der Brief Hamanns an Kant 27. Juli 1759 (Hamanns Schriften I. $429 \mathrm{ff}$.), in welchem das innere Verhältnis der beiden zum ersten Mal deutlich zu Worte kommt. Denselben Zweck, sich innerlich mit dem Bekehrungsversuch Kants, vor allem freilich mit Berens, auseinanderzusetzen, haben die "Sokratischen Denkwürdigkeiten“. Hamann schreibt darüber am 12. Oktober 1759 an Lindner: „Ja, dies ist meines Herzens Wunsch, mit dem Magister Kant nicht paragraphenweise, sondern das Ganze, was man geschrieben und gelebt, zu überlegen, damit das Tumultuarische nicht das Gute ersticke." Die Schrift wurde in den Hamburgischen Nachrichten in gehässiger Weise besprochen. Der Allnahme, dass diese Recension direkt oder indirekt anf Kant zurückgehe, oder das wenigstens Hamann dies angenommen habe, widerspricht Weber (Seite 34). Übrigens findet sich in dieser Recension der recht oberflächliche Vergleich Hamanns mit Jakob Böhme.

In dasselbe Jahr 1759 fällt noch ein Brief Hamanns an Kant, über dessen Plan, mit Hamann gemeinsam eine Physik für Kinder zu schreiben. Hamann soll offenbar für die populäre und kindliche Form sorgen. Die Absage Hamanus liegt vor in einem Brief an Kant (Schriften I, 504 ff.) und in „der Zugabe zweener Liebesbriefe an einen Lehrer der Weltweisheit, der eine Physik für Kinder schreiben wollte ${ }^{u}$. Aus dem Briefwechsel wird deutlich, dass das Zusammenarbeiten beider freilich eine innere Unmöglichkeit ist.

Ebenso deutlich tritt die Eigenart beider Männer in dem Briefwechsel über Herders, „älteste Urkunde" hervor. Während Kant lediglich wissen will, ob er in einigen präzisen Sätzen Herder richtig verstanden hat, ist es Hamann unmöglich, der sehr bezeichuenden Bitte Kants za entsprechen: „Ich bitte mir Ihre 
Meinung in einigen Zeilen aus; aber wonıglich in der Sprache dor Menschen. Deun ich armer Erdensolun bin zur Gottersprache der anschauenden Vernunft gar niclit organisiert. Was man mir aus den gemeinen Begriffen nach logischer Regel vorbnchstabieren kann, das erreiche ich noch wohl." Freilich bekommt Hamann auf seine Antwort von Herder das Zeugnis: "Sie haben meinen Sinn und Zweck nicht bloss wohl gefasst, sondern anch sehr gesäubert und idealisiert, dass in der Folge mir Ihre Winke auf meiner Bahn zu Hilfe kommen werden, dass ich ein reineres und sicheres Ziel nehme.... Mich freut sehr, dass sonderlich Ihr Anfang so hell geworden; wer das nicht versteht, dem kann niemand belfen." Gleichwohl begreift man, dass Kant, der beiden ferner stand, anders urteilt. Nur über Hamanns „Apologie des

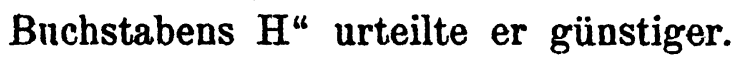

Aus dem späteren Verkehr beider haben für die Biographie Kants Interesse die Verhandlungen über den Verleger „der Kritik der reinen Vernunft" (Seite $64 \mathrm{ff}$.). Dazu ist aus der neueren Veröffentlichung der Brief, Seite 79, an Hartknoch zu vergleichen. Hier findet sich auch noch ein Brief, der den unmittelbaren Eindruck der ersten Lektüre der Kritik wiedergiebt (Seite $80 \mathrm{ff}$.).

Das äussere Verhältnis beider Männer zu einander beschreibt Weber, Seite 74,' so: „Die persönliche Verbindung war in den letzten Lebensjahren des Magus recht lose; es fehlte die Gegenseitigkeit. Hamanns Teilnahme ist nicht erkaltet. Er nahm „nicht bloss als Landmann und Patriot, sondern aus weit näheren Interesse an Kants Autorschaft Anteil", wie er im Mai 1786 schreibt. Die Erwiderung der Anteilnahme seitens Kants blieb aus. Hamann ist orientiert über Kants Arbeiten, bringt ihm auch litterarische Neuheiten. Die Höflichkeit der gegenseitigen Schriftenzusendung wurde auch jetzt nicht unterlassen." Kant hatte dem bedrängten Hamann früher schon einmal zu einer Anstellung verholfen (Seite 51). Im allgemeinen aber kommt Weber in Unterschied von Gildemeister zu dem Resultat, dass die Freundschaft eine einseitige war. Von Hamanns Seite war sie aufrichtig, Kant hat sie nicht wirklich erwidert (Seite 85 und 104).

Das hatte nicht nur äusserliche Gründe, sondern ergab sich aus dem innersten Wesen und Denken heider Männer. Kants Kühle und die Vereinigung von persốnlicher Freundschaft und prinzipiellem Widerspruch auf Hamanns Seite erklärt sich völlig* aus der philosophischen und religiösen Eigenart beider. Wieder- 
holt spricht es Hamann aus, dass Kant, je länger desto mehr, in die Eigenart aller Systematiker verfalle, alle Menschen nach ihrer Stellung zu seinem System zu beurteilen. Widerspruch und Kritik kann er nicht vertragen. In andere Menschen oder Gedankengänge sich hinein zu versetzen, ist ihm unmöglich. Darum ist er auch ein schwacher Historiker. Die Selbstrerlengnung, die zur Geschichtsschreibung gehört, ist ihm unmöglich. Hamaun 1) dagegen ist nicht nur kein Systematiker, sondern er meidet und hasst alles systematisieren. Nicht etwa aus persönlichem Unvermögen, sondern mit voller Absichtlichkeit. Sein Wirklichkeitssinn sträubt sich dagegen. Die Wirklichkeit lässt sich in keiu System fassen, unser Wissen ist Stïckwerk und eben darum darf es durch die Form eines Systems nicht den Schein eines Ganzen erwecken. Wir kennen und verstehen einzelnes, aber nicht alles und darum ist unser Wissen kein Ganzes. - Daraus erklärt sich sein Styl. Er hat ihn selbst bald als Last empfunden, bald als Vorzug gepriesen. Er entspricht seinem Denken. Er ist aphoristisch, sprunghaft. Hamann vermag nicht und versucht auch nicht, die logischen Bindeglieder zwischen den einzelnen Gedanken hervorzuheben, denn er ist nie durch einen Schluss vom einen zum anderen Gedanken gekommen. Jeder ist eine Beobachtung für sich und stammt nicht aus einem.Schluss, sondern aus einer Wahrnehmung. Alle unsere Erkenntnisse, auch die mathematischen, bekommen wir durch die Vermittelung unserer fünf Sinne. Hamann ist Empirist im höchsten Masse. Formell treten daher seine Gedanken als Einfälle auf, blitzartig, ohne Zusammenhang unter einander. Für $i_{\text {hn }}$ selbst sind sie Inspirationen, göttliche Eingebungen, denn die Wirklichkeit, aus der er sie intuitiv schöpft, 'ist ihm eine Offenbarung Gottes. Er liebt daher die Divination (Unger S. 117) und das Denkeu aus dem Ganzen, die Intuition im. Gegensatz zur Induktion (Unger S. 95). Wer Kant kennt, begreift, dass er für ihn ein Schwärmer sein musste. "Zum guten Teil auf Hamann zielen die Reflexionen über die Schwärner, wie schon B. Erdmanu gezeigt hat" (S. 98, 112, 169 ff.). Kant stellt inn neben Jakob Bölume und Swedenborg. Beides freilich schon äusserlich mit Unrecht. Von Jakob Böhme hat Hanaun nur wenig gewusst. Über

1) Eine präzise plastische Charakteristik Hamanns und seines Denkens von seinem Styl aus giebt Unger S. 24-44. Zuweilen scheint mir, wird er in dem berechtigten Bestreben, die kritikluse Begeisterung der Anhänger Hamanns zu korrigieren, ihm doch nicht ganz gerecht. 
Swedenborg, dessen Werke er sich übrigens von Kant geliehen hatte, urteilt er recht scharf (Weber S. 270 f.). Eine innerliche Berührung mit Jakob Böhme lăsst sich nur an einem Pankte erkennen, nämlich ill dem von Hamann so hoch gestellten principium coincidentiae oppositornm. Dies spielt freilich nicht in der Logik, aber in der Willenslehre Böhmes eine grosse Rolle. Und es ist möglich, dass hier Zusammenhänge bestehen, die Hamann selbst nicht zum Bewusstsein gekommen sind. Jedenfalls ist es jedem, ler Kant kennt, klar, dass ihn Hamanns Schwärmerei für Widersprüche ein Greuel seil musste. Dieser Zug gehört zu Hamanns Wirklichkeitssinn. Er dachte in dieser Beziehung über seine eigene Gedankeuwelt ganz wie Goethe: „Die Welt ist voller Widerspruch und sollte sichs nicht widersprechen". Wenn Kant ihn auch im religiösen Sinne einen Schwärmer nennt, so ist das jedenfalls ungerecht; denn wenn man nicht alle ernsthafte Theologie als Schwärmerei beurteilt, sondern unter religiöser Schwärmerei den Versuch versteht, den Standpunkt des Glaubens durch eine innere mystische Erfahrung zu überbieten, so war Hamann jedenfalls kein Schwärmer (Weber S. 174), sondern teilte in dieser Beziehung das Urteil Luthers über die Schwärmer.

Es ist begreiflich, dass Kant durch kein Urteil Hamanns mehr verstimmt wurde, als dadurch, dass er ihm gerade Mystik vorwarf, denn von nichts fülulte sich Kant weiter entfernt (Weber S. 70 und 214). Hamann begründet den Vorwurf so: "das Sprechen in lauter Begriffen, denen die entsprechenden anschaulichen Objekte fehlen, also in abstrakten Begriffen, dies macht die Philosophen zu mystischen Schwärmern. Weit entfernt vom Boden der greifbaren Wirklichkeit irren sie in den Höhenregionen abenteuerlich aumutender Begriffe umher". Seinem "naiven Realismus “ war Kants transscendentaler Idealismus Mystik.

Im ganzen jedoch hatte Hamaun für Kant persönlich ein weit grösseres Verständnis, als Kant für ihn. Freilich spricht er in seinen Briefen an Kant gelegentlich mit einem Selbstbewusstsein, dass mit Kants nicht geringerem Selbstbewusstsein kräftig zusammenstiess. Allein man muss ihn kennen, um das zu verstehen. Selbstbewusstsein und Demut vereinigte er wie in seiner Theorie, so in seiner persönlichen Haltung in eigentümlicher Weise mit einander. Er spricht das sehr deutlich in den Briefe an Kant I, $504 \mathrm{ff}$. ans. Fbensowenig darf man, wie mir in Übereinstimnung mit. Weber scheint, seine Urteile über Kant als iro- 
nisch und darum als nicht ernst gemeint ansehen, wie das der Herausgeber dieser Zeitschrift gethan hat. Nan muss Hamanns Humor kennen, den er auch gegen sich selbst wendet, wenn man seine Urteile verstehen will. Er hat die Bitterkeit und Empfindlichkeit Herders Kant gegenüber nicht gebilligt; sondern zu mildern versucht und bat Herder gegenüber das relative Recht Kants vertreten.

Trotz alledem ist natürlich die innere Differenz zwischen Hamann und Kant gross, und zwar um so grösser, als sie religiös begründet ist. In welchem Masse Hamanns gesamte Gedankenwelt in seiner Bekehrungsgeschichte begründet ist, hat besonders Unger treffend hervorgehoben. ${ }^{1}$ ) Hamann ist in dieser Beziehung durchaus mit Paulus, Augustin und Luther zu vergleichen. Auch seine erkenntnistheoretischen Bedenken gegen Kants Kritik sind religiös begründet. Die grundsätzliche Ausscheidung des Gottesgedankens aus der Naturforschung und Philosophie, die Kant vollzog, widersprach dem Gottesbewusstsein Hamanns aufs schärfste, denn für ihn sind Natur und Geschichte durchsichtige, symbolische Darstellungen des göttlichen Willens. Freilich fühlte er sich den Kritizismus Kants insofern verwandt, als er, wie Kant von Hume ausging. Kant ist ihm der "preussische Hume“. Allein der Anschluss an Hume hat doch bei beiden sehr verschiedenen Grund. Hamann steht wie Kant der Vernunft kritisch gegenüber, allein seine skeptischen Urteile über den Wert der menschlichen Vernunft haben einen ganz anderen Sinn als Kants Kritik. Sie schliessen sich an Luthers bekannte Urteile und über ihn zurück an das Urteil des Paulus an. Für ihn ist seine radikale Skepsis, verbunden mit seiner sensualistischen Erkenntnistheorie, ganz ähnlich wie - für Pascal, lediglich die Voraussetzung seiner Glaubensphilosophie. Dagegen ist Glaube für ihn eine Erkenntnis, die über die der Vernunft gesetzten Schranken hinansgreift, eben weil alle Erfahrung für ihn den Charakter der Offenbarung trägt, d. h. Kenntnis des jenseitigen Gottes vermittelt (Weber S. 185 ff.). Mit diesem positiven Urteil schied er sich uatürlich von Kants Kritik.

1) Doch ist Unger - begreiflicherweise - von den theologischen Autorităten, die er bei seiner umfassenden Litteraturbenutzung ebenfalls reichlich herangezogen hat, zuweilen zu abhängig. Ich kann deshalb nicht alle seine Urteile toilen. 
Elhenso fühlte er sich in der Ethik ron Kant geschieden. Denu Kants Lelıre vom radikalun Bösen ist ja etwas ganz anderes, als der vou deu Reformatoren stammende moralische Pessimismus Hamanus (S. $123 \mathrm{ff}$. . In dem Schluss: "Da kannst, denn du sollst" liegt fiir Hamaun ein Optimismns, der durch die Erfabruug niderlegt wird.

Am eigentiimlichsten sind die pusitive ॥ Sätze, die Hamann ler Kritik der reinen Vernunft entgegenstellt. Freilich ist ibm ja nauches Nissverständnis untergelaufen (Veber S. $223 \mathrm{ff}$.). Schon den Ausdruck ${ }_{n}$ reine" Vernunft nissverstand er; indem er ihn neben dell anderen Ausdruck "guter Wille“ stellt, bemerkt man, was übrigens auch aus seiner Polemik hervorgeht, dass er in diesem Ausdruck eine Verneinung des reformatorischen Gedankens sah, dass die Vernunft verderbt und damit zur Erkenntnis Gottes unfähig sei. Ebensowenig ist ihm der Unterschied Kants vom Rationalismus klar geworden. Den Apriorismus Kants missverstand er völlig, indem er ihn dahin deutete, dass der Meusch aus sich heraus, ex vi formae der Venunft, ohne Erfahrung, d. h. also in Hamanns Ausdruckweise übersetzt, „ohne Offenbarung “ etwas erkennen könne (Unger S. 101). Gleichwohl ẹthält seine Kritik Kants bedeutsame Gedanken. Sie steht in Zusammenhang mit seiner Theorie der Sprache, die Unger ausführlich und gründlich aus dem Zusammenhang der Weltanschauung Hamanns erklärt und in ihren geschichtlichen Zusammenhängen und Wirkungen dargestellt hat. Er hat dabei die Gefahr der Freunde Hamanns, unter allen Umständen System und Einheit in die Gedankenwelt Hamanns zu bringen, sorgfältig und zuweilen eher etwas zu ängstlich vermieden.

Hamann protestiert zunächst gegen den Kantischen Dualismus von Sinnlichkeit und Verstand. Beide sind geeinigt in der Sprache. Das Wort ist die Einheit von Anschauung und Begriff. Vernunft ist Sprache. Das ist sein immer wiederkehrender Grandsatz. Er hat als der erste in Deutschland (Unger S. 214) jedenfalls das Verdienst, über das Verhältnis von Sprechen und Denken nachgedacht und darauf hingewiesen zu haben, dass eine Lehre von der Vernunft ohne eine Theorie der Sprache eine Unmöglichkeit ist. Anf der Sprache beruht für ihn nicht nur die Möglichkeit des Denkens, sondern auch die Probleme, die unvermeidlichen Täuschungen, die die Kritik der reinen Vernunft als solche erkenuen lehren wollte, beruhen auf den. Hypostasierungen und 
Personifikationen, die besouders uurch den Gebrauch des Substantivs in die Sprache kommen: Kritik der reinen Vernunft ist Kritik der Sprache. Dass dies ein furchtbarer Gedanke war, ist nicht zu bezweifeln, wenn man auch vielleicht Webers Urteil nicht teilt. „Hamann hat die Entwickelung anticipiert, die von der Transscendentalphilosophie und ihrer aprioristischen Erkenntnislehre hinweg zu der psychologischen Lösung des Erkenntnisproblems hindrängte.“

Zum Schluss möchte ich nur bemerken, dass nicht nur die allgemeinen religiösen Grundgedanken Hamanns ihre Wurzel bei Luther haben, sondern dass auch einige seiner speziellen Ideen daraufhin untersucht werden mussten, ob und inwieweit sie von Luther stanmen.

Dass wir Veruunft nur in Form der Sprache haben, entspricht und entspringt vielleicht auch dem Grundsatz Luthers, dass wir den Geist nur im Worte haben. Auf Luther weist auch der Begriff der conmunicatio idiomatum des Göttlichen und Menschlichen (Unger S. 237) und der Wert, den Hamann diesem Grundgesetz beilegt, hin, ebenso der Ausdruck, dass die Einheit von Sinnlichem und Begrifflichem im Wort eine sakramentale sei. Vielleicht lohnt es sich, zu untersuchen, inwieweit Hamann von Luther gelernt hat.

Doch das gehört nicht hierher. Für uns handelt es sich nur um sein Verhältnis zu Kant. 